\title{
LEFT VERSUS RIGHT LCM DOMAINS
}

\author{
RAYMOND A. BEAUREGARD
}

\begin{abstract}
It is well known that every right Bezout domain satisfying the left Ore (multiple) condition is a left Bezout domain. A similar statement for the smaller class of principal right ideal domains is a long-standing conjecture which remains unresolved. In this paper we settle the analogous question for the larger class of right LCM domains.
\end{abstract}

This paper deals with the question of the left-right symmetry of a right LCM domain, i.e. an integral domain in which the intersection of two principal right ideals is again principal. In the smaller class of right Bezout domains it is known that these are left Bezout domains provided it is assumed that they satisfy the left Ore condition: $R a \cap R b \neq 0$ for all nonzero $a, b$ in $R$. This follows from the fact that the definition of a weak Bezout domain (also known as a 2-fir) is left-right symmetric [3]. In an even smaller class the question of whether a left Ore PRI (principal right ideal) domain is a PLI domain remains open.

Below it is shown that a left Ore right LCM domain need not be a left LCM domain in general but will be under the additional hypothesis that the ring has the ascending chain condition for principal left ideals. In fact the example given is that of a left and right bounded right LCM domain which is not a left LCM domain. This stands in contrast to the corresponding result that a left bounded PRI domain is a PLI domain [2].

In what follows $R$ denotes a ring with unity and without proper divisors of zero; $R^{*}$ denotes the monoid of nonzero members of $R$. For $x \in R^{*}$ let $[x R, R]$ be the set of principal right ideals of $R$ that contain $x$. Note that each $[x R, R]$ is partially ordered by inclusion.

Proposition 1. For each $x$ in $R^{*}$ the intervals $[x R, R]$ and $[R x, R]$ are dually isomorphic; in particular if $x=a a^{\prime}$ then the correspondence $a R \leftrightarrow R a^{\prime}$ is a bijection which reverses order.

Proof. Let $x=a a^{\prime}=b b^{\prime}$. Then $a R \subseteq b R$ iff $a=b c$ for some $c$ in $R$, and this is so iff $b^{\prime}=c a^{\prime}$ for some $c$ in $R$, i.e. iff $R b^{\prime} \subseteq R a^{\prime}$. Note that either containment becomes equality iff $c$ is a unit in $R$.

Proposition 2. If the interval $[x R, R]$ is a lattice for each $x$ in $R^{*}$ and if $R$ is $a$ right Ore domain then $R$ is a right LCM domain.

Received by the editors February 6, 1979.

AMS (MOS) subject classifications (1970). Primary 16A02.

Key words and phrases. Right LCM domain, left-right symmetry, intervals of principal right ideals, (left) Ore condition. 
Proof. Let $a, b \in R^{*}$ and suppose $0 \neq x_{i} \in a R \cap b R$. Let $m_{i} R=a R \wedge_{i} b R$, the meet of $a R$ and $b R$ in $\left[x_{i} R, R\right](i=1,2)$. We proceed to show $m_{1} R=m_{2} R$. Let $0 \neq z \in m_{1} R \cap m_{2} R$ (using the right Ore condition) and let $m_{z} R=a R \wedge_{z} b R$ in $[z R, R]$; thus $m_{i} R \subseteq m_{z} R$. But then $x_{i} R \subseteq m_{i} R \subseteq m_{z} R \subseteq a R \cup b R$ implies $m_{i} R$ $=m_{z} R$, i.e. $m_{1} R=m_{2} R$. Proposition 2 now follows immediately.

THEOREM. Let $R$ be a right LCM domain. If $R$ has the ascending chain condition for principal left ideals and satisfies the left Ore condition then $R$ is a left LCM domain.

Proof. Let $x \in R^{*}$. Since $[R x, R]$ has the acc by hypothesis, $[x R, R]$ has the dcc by Proposition 1. Since $R$ is a right LCM domain $[x R, R]$ is a meet semilattice which by the dcc must be a lattice. It follows by Proposition 1 that the interval $[R x, R]$ is a lattice for each $x$ in $R^{*}$. Applying the left-right analog of Proposition 2 we conclude that $R$ is a left LCM domain.

The following is an example of a bounded (hence left Ore) right LCM domain which is not a left LCM domain.

EXAMPLe. Let $K$ be a local PRI domain which is not a PLI domain (for example (cf. [4]) $K=L[[t, \sigma]]$ where $a t=t a^{\sigma}$ and $\sigma$ is a monomorphism on the field $L$ which is not an isomorphism). Let $F=K\left(K^{*}\right)^{-1}$, the quotient field of $K$, and let $P=F[[x]]$, the ring of formal power series in the central indeterminate $x$. The example we seek is the following subring of $P: R=\{f(x) \in P \mid f(0) \in K\}$.

(i) $R$ is a right LCM domain.

Let $f, g \in R$. We might as well assume that $f R \nsubseteq g R, g R \nsubseteq f R$, and $\operatorname{ord}(g)<$ $\operatorname{ord}(f)$. Thus $f=g h_{0}$ for some $h_{0} \in P$ and we may choose $d \in K$ such that $f d \in g R$ (note that $d$ is not a unit by the assumptions above). In fact, we choose such a $d$ for which $d K$ is maximal ( $K$ has the acc for right ideals). We have $f d R \subseteq f R \cap g R$. To show the reverse inclusion let $f h_{1}=g h_{2}\left(h_{i} \in R\right)$. Then $f h_{1}=f d h_{3}$ for some $h_{3} \in P$ (since $f P \cap g P=f d P=f P$ ). If $h_{3}(0)=0$ then $h_{3} \in R$ and $f h_{1} \in f d R$. If $h_{3}(0) \neq 0$ then writing $h_{1}=a u$ where $u \in R$ and $u(0)=1$ we find $f a=f h_{1} u^{-1}=g\left(h_{2} u^{-1}\right) \in g R$. Comparing $d K$ with $a K$ in $K$ we have either $a K \subseteq d K$ or $d K \subsetneq a K$, the latter choice being impossible because of the maximal nature of $d K$. Thus $a=d c$ for some $c \in K$ and $f h_{1}=f a u=f d(c u) \in f d R$ as desired.

(ii) $R$ is not a left LCM domain.

Recalling [1] that in an integral domain if $0 \neq\left(x a^{-1}\right) a=\left(x b^{-1}\right) b$ then existence of the $\operatorname{lclm}[a, b]_{1}$ implies existence of the hclf $\left(x a^{-1}, x b^{-1}\right)_{1}$, it suffices to show that the latter does not exist in $R$. Suppose then that $h=\left(x a^{-1}, x b^{-1}\right)_{1}$ in $R ; h$ may be written $h=x^{n} s u$ where $n=0$ or $1, s \in F$, and $u(0)=1$. Since $u$ is a unit in $R$ we may omit it; since $x a^{-1}, x b^{-1} \in t R$ for all $t$ in $K^{*}, h$ must not be in $K$. Thus $h=x s$. We have $x a^{-1}=x s r_{1}, x b^{-1}=x s r_{2}$ for some $r_{i} \in K$ so that $s=a^{-1} r_{1}^{-1}=$ $b^{-1} r_{2}^{-1}$ and $r_{1} a=r_{2} b \neq 0$. This argument shows that if $R$ is a left LCM domain then $K$ must be left Ore; but $K$ is not left Ore, for otherwise $K$ would be a PLI domain by [2, Corollary 1].

(iii) $R$ is a left Ore domain. 
This follows immediately from the fact that $P$ is a left Ore domain (being a PLI domain). More specifically we show that $R$ is left bounded: if $f=x^{n} u a b^{-1}$ where $a, b \in K$ and $v \in R$ with $v(0)=1$ then $x^{n+1}=\left(x b a^{-1} u^{-1}\right) f \in R f$, i.e., $R f$ contains the two-sided ideal $R x^{n+1}$. Similarly $R$ is right bounded.

\section{BIBLIOGRAPHY}

1. R. A. Beauregard, Right LCM domains, Proc. Amer. Math. Soc. 30 (1971), 1-7.

2. __Left and right invariance in an integral domain, Proc. Amer. Math. Soc. 67 (1977), 201-205.

3. P. M. Cohn, Free rings and their relations, Academic Press, London, 1971.

4. A. V. Jategaonkar, Left principal ideal domains, J. Algebra 8 (1968), 148-155.

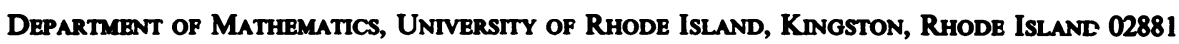

